\title{
Moderate Esophageal Dysplasia
}

National Cancer Institute

\section{Source}

National Cancer Institute. Moderate Esophageal Dysplasia. NCI Thesaurus. Code C4841.

A morphologic finding indicating the presence of moderate dysplastic cellular changes and moderate architectural changes in the epithelium of the esophageal mucosa. There is no evidence of invasion. 